\title{
Ferulic acid prevents liver injury and increases the anti-tumor effect of diosbulbin B in vivo*
}

\author{
Jun-ming WANG ${ }^{1,3}$, Yu-chen SHENG ${ }^{2}$, Li-li JI ${ }^{\dagger+1}$, Zheng-tao WANG ${ }^{1}$ \\ $\left({ }^{1}\right.$ MOE Key Laboratory for Standardization of Chinese Medicines and Shanghai Key Laboratory of Complex Prescription, \\ Institute of Chinese Materia Medica, Shanghai University of Traditional Chinese Medicine, Shanghai 201203, China) \\ $\left({ }^{2}\right.$ Center for Drug Safety Evaluation and Research, Shanghai University of Traditional Chinese Medicine, Shanghai 201203, China) \\ ( ${ }^{3}$ School of Pharmacy, Henan University of Traditional Chinese Medicine, Zhengzhou 450008, China) \\ †E-mail: lichenyue1307@126.com
}

Received Sept. 16, 2013; Revision accepted Mar. 17, 2014; Crosschecked May 22, 2014

\begin{abstract}
The present study is designed to investigate the protection by ferulic acid against the hepatotoxicity induced by diosbulbin B and its possible mechanism, and further observe whether ferulic acid augments diosbulbin Binduced anti-tumor activity. The results show that ferulic acid decreases diosbulbin B-increased serum alanine transaminase/aspartate transaminase (ALT/AST) levels. Ferulic acid also decreases lipid peroxide (LPO) levels which are elevated in diosbulbin B-treated mice. Histological evaluation of the liver demonstrates hydropic degeneration in diosbulbin B-treated mice, while ferulic acid reverses this injury. Moreover, the activities of copper- and zinc-containing superoxide dismutase (CuZn-SOD) and catalase (CAT) are decreased in the livers of diosbulbin B-treated mice, while ferulic acid reverses these decreases. Further results demonstrate that the mRNA expressions of CuZn-SOD and CAT in diosbulbin B-treated mouse liver are significantly decreased, while ferulic acid prevents this decrease. In addition, ferulic acid also augments diosbulbin B-induced tumor growth inhibition compared with diosbulbin B alone. Taken together, the present study shows that ferulic acid prevents diosbulbin B-induced liver injury via ameliorating diosbulbin B-induced liver oxidative stress injury and augments diosbulbin B-induced anti-tumor activity.
\end{abstract}

Key words: Ferulic acid, Diosbulbin B, Hepatotoxicity, Oxidative stress injury, Anti-tumor activity doi: 10.1631 jzus.B1300250

Document code: A

CLC number: R932

\section{Introduction}

Dioscorea bulbifera L. (Dioscoreaceae) rhizome is widely distributed throughout the tropics and temperate regions, especially in Asia, and in China, it has been traditionally used to treat thyroid disease and tumors (Gao et al., 2002). In addition, in northern Bangladesh, it is used to treat tumors and leprosy (Murray et al., 1984). Our laboratory studies have shown that D. bulbifera rhizome can induce hepato-

\footnotetext{
Corresponding author

* Project supported by the Program for New Century Excellent Talents in University (No. NCET-11-1054), the National Natural Science Foundation of China (No. 81322053), and the Program for Changjiang Scholars and Innovative Research Team in University (No. PCSIRTIRT1071), China

(C) Zhejiang University and Springer-Verlag Berlin Heidelberg 2014
}

toxicity (Wang et al., 2010; 2011), which emphasizes a potential toxicity in the clinical setting. Among the biologically active substances in $D$. bulbifera rhizome, a major class is the clerodane diterpenoids (Wang et al., 2009; Liu et al., 2010). Diosbulbin B is the major chemical compound of the clerodane diterpenoids in D. bulbifera rhizome (Kawasaki et al., 1968), and has been found to have anti-tumor activity (Gao et al., 2002; Wang et al., 2012).

Angelica sinensis (Oliv.) Diels root has been reported to prevent the hepatotoxicity induced by $D$. bulbifera rhizome (Liu et al., 2004), while the active compound has not yet been elucidated. Ferulic acid is one of the main bioactive molecules in $A$. sinensis root, and always acts as a strong anti-oxidant; it is known for treating skin cancer, ageing, fatigue, and 
muscle wasting (Graf, 1992; Alias et al., 2009; Barone et al., 2009; You et al., 2009). There are reports that ferulic acid prevents noise-induced hearing loss and free radical-induced skin damage (Calabrese et al., 2008; Fetoni et al., 2010), but whether it affects the hepatotoxicity and anti-tumor activity of diosbulbin $B$ is still unknown.

Various reports have demonstrated that oxidative stress plays a vital role in liver toxicity induced by such substances as alcohol, carbon tetrachloride, chemotherapeutic agents, and acetaminophen (dos Santos et al., 2007; Kostopanagiotou et al., 2009; Moselhy and Ali, 2009; Samuhasaneeto et al., 2009). Reactive oxygen species (ROS), generated during oxidative stress, are extremely reactive and may modify and inactivate cellular lipids, proteins, DNA, and RNA, and thus cause cell damage. The cellular anti-oxidant system in the body prevents ROS-induced cell injury; the system includes low-molecular-mass anti-oxidants (van der Vliet et al., 1999) such as glutathione, $\alpha$-tocopherol, ascorbic acid, and the main anti-oxidant enzymes including copper- and zinccontaining superoxide dismutase (CuZn-SOD) and catalase (CAT). Our previous report demonstrated that oxidative stress plays an important role in liver injury induced by acetate extracts of $D$. bulbifera (Wang et al., 2011); however, whether the protection by ferulic acid against liver injury induced by diosbulbin $B$ is related to the liver anti-oxidant system has not been demonstrated.

The present study is designed to demonstrate the protection by ferulic acid against the hepatotoxicity induced by diosbulbin $\mathrm{B}$ and the underlying mechanism, and investigate whether ferulic acid enhances diosbulbin B-inhibited tumor growth.

\section{Materials and methods}

\subsection{Experimental animals}

ICR male mice (18-22 g) were purchased from the Shanghai Laboratory Animal Center of Chinese Academy of Sciences, China. Animals were given rodent laboratory chow and water ad libitum, and maintained under controlled conditions at $(22 \pm 1){ }^{\circ} \mathrm{C}$, relative humidity $(65 \pm 10) \%$, and a $12 \mathrm{~h} / 12 \mathrm{~h}$ light/ dark cycle (lights on at 7:00 am). All animals were dealt with in compliance with the institutional animal care guidelines of the Experimental Animal Ethical Committee of Shanghai University of Traditional Chinese Medicine, China.

\subsection{Cell lines}

Mouse sarcoma S180 tumor cells were maintained in the peritoneal cavities of ICR mice in the Laboratory of Experimental Oncology of the Shanghai Laboratory Animal Center of Chinese Academy of Sciences, China.

\subsection{Reagents}

5-Fluorouracil was purchased from Shanghai Bangcheng Chemical Co., Ltd., China. Unless indicated, other reagents were purchased from Sigma Chemical Co. (St. Louis, MO, USA).

\subsection{Preparations of diosbulbin $B$ and ferulic acid}

D. bulbifera rhizome was collected in Qingyang County, Anhui Province, China and authenticated by Prof. Shou-jin LIU (Anhui University of Chinese Medicine, China). A. sinensis root was purchased from Minxian County, Gansu Province, China and authenticated by Prof. Li-hong WU (Shanghai University of Traditional Chinese Medicine, China). The voucher specimens of $D$. bulbifera rhizome and A. sinensis root were both deposited in the herbarium of the Institute of Traditional Chinese Medicine, Shanghai University of Traditional Chinese Medicine, China.

Diosbulbin B and ferulic acid were isolated from $D$. bulbifera rhizome and A. sinensis root, respectively, in our laboratory as previously described (Kawasaki et al., 1968; Sun et al., 2006). After purification using silica gel column and gel chromatography, purities of diosbulbin $\mathrm{B}$ and ferulic acid were both more than $98 \%$ as determined by high-performance liquid chromatography (HPLC) with diode array detector. The chemical structures of diosbulbin B and ferulic acid are shown in Fig. 1.

\subsection{Treatment protocol}

Ascites of the mouse sarcoma S180 were drawn out from mice under aseptic conditions. Cells were counted promptly and then diluted to about $1 \times 10^{7}$ cells $/ \mathrm{ml}$ with aseptic saline. The diluted S180 cell suspension was inoculated subcutaneously $(0.1 \mathrm{ml}$ per mouse $)$ into the right armpit (Bezerra et al., 2008). 
(a)

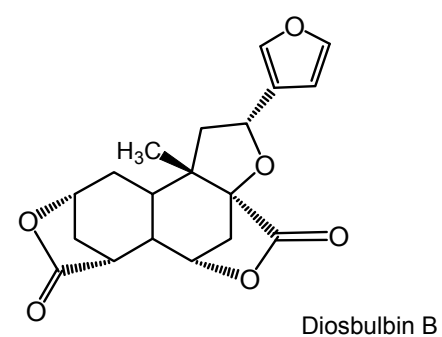

(b)

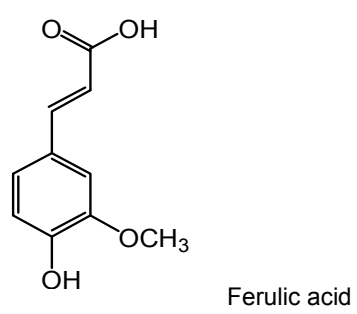

Fig. 1 Chemical structures of diosbulbin B (a) and ferulic acid (b)

One day after inoculation, mice, except for the normal (non-tumor-inoculated) animals, were randomly divided into five groups of eight mice each. 5-Fluorouracil-treated mice were injected intraperitoneally with 5 -fluorouracil at a dose of $25 \mathrm{mg} / \mathrm{kg}$ once every other day. Normal (non-tumor-inoculated) and control (tumor-inoculated) groups of mice received daily oral administration of $0.5 \%(5 \mathrm{~g} / \mathrm{L})$ sodium carboxyl methyl cellulose (CMC-Na; $0.2 \mathrm{ml} / 10 \mathrm{~g}$ ). The other three groups received ferulic acid $(8 \mathrm{mg} / \mathrm{kg})$, diosbulbin B (32 mg/kg), or diosbulbin B (32 mg/kg) plus ferulic acid $(8 \mathrm{mg} / \mathrm{kg})$ by intragastric administration for $12 \mathrm{~d}$ starting from $24 \mathrm{~h}$ after tumor inoculation. After treatment, mice were sacrificed by cervical dislocation after peripheral blood samples, liver tissues and tumors were collected at $24 \mathrm{~h}$ after the last administration. Serum samples were collected for the determination of alanine transaminase/aspartate transaminase (ALT/AST), and liver tissues were used for the analysis of the lipid peroxide level, histological observation, determination of glutathione-related and anti-oxidant enzymes, and mRNA expression of the main anti-oxidant enzymes. The tumors were weighed, arrayed in line on paper, and taken pictures. The tumor inhibition ratio (IR) was calculated by the formula of $\mathrm{IR}=[(C-T) / C] \times 100 \%$, where $C$ and $T$ are the mean tumor weights of the control group (CMC-Na) and the treated group, respectively.

\subsection{Assay for serum ALT and AST}

Blood samples were obtained from mice of all groups (eight mice per group) for the determination of ALT and AST. Serum ALT and AST were assayed according to Kamei et al. (1986).

\subsection{Assay for liver lipid peroxide level}

Liver tissues were homogenized in cold phosphate buffered saline (PBS). Lipid peroxide was determined as previously described (Nkosi et al., 2006). Malondialdehyde is an end product of lipid peroxide and serves as a means of quantifying lipid peroxide. Malondialdehyde reacts with 2-thiobarbituric acid to generate a pink-colored product, which has an absorbance at $532 \mathrm{~nm}$. The standard curve of malondialdehyde was constructed over the concentration range of $0-40 \mu \mathrm{mol} / \mathrm{L}$. Lipid peroxide level was expressed as micromoles of malondialdehyde per milligram of protein based on tissue protein concentration.

\subsection{Histological observation}

After fixation in formaldehyde, the livers were examined for size, color changes, and hemorrhage. Slices of liver were cut into small pieces and histological sections were stained with hematoxylin and eosin (H\&E) for the observation under light microscopy.

\subsection{Enzymatic assays}

Tissues were homogenized in cold PBS, and then centrifuged at $5000 \times g$ for $5 \mathrm{~min}$ and the supernatant transferred to new tubes for assay. The liver tissue activities of $\mathrm{CuZn}$-SOD and CAT were determined as previously described (Marklund and Marklund, 1974; Aebi, 1984), and the results were all calculated based on tissue protein concentrations.

\subsection{Reverse transcription-polymerase chain re- action (RT-PCR) analysis}

Total RNA was extracted from the liver tissue using Trizol (Life Technologies, USA) according to the manufacturer's protocol. To synthesize singlestrand cDNA, reverse transcription of $2 \mu \mathrm{g}$ total RNA was carried out using 200 U Moloney murine leukemia virus reverse transcriptase (M-MLV RT) and reaction mixture $(2.5 \mathrm{mmol} / \mathrm{L}$ dNTP, $100 \mathrm{pmol} / \mathrm{L}$ oligo(dT) primer, RT buffer, 50 U RNA inhibitor) according to the manufacturer's protocol. Transcripts 
of the gene for glyceraldehyde-3-phosphate dehydrogenase (GAPDH) were used as an internal control. Sequences of the PCR primers were as follows: CuZn-SOD forward 5'-AAG GCC GTG TGC GTG CTG AA-3', reverse 5'-CAG GTC TCC AAC ATG CCT CT-3' (246 bp product) (El Mouatassim et al., 1999); CAT forward 5'-GCA GAT ACC TGT GAA CTG TC-3', reverse 5'-GTA GAA TGT CCG CAC CTG AG-3' (229 bp product) (El Mouatassim et al., 1999); and, GAPDH forward 5'-GAC CCC TTC ATT GAC CTC AAC T-3', Reverse 5'-GTT TGT GAT GGG TGT GAA CCA-3' (200 bp product) (Hougardy et al., 2005). The PCR protocol consisted of denaturation at $94{ }^{\circ} \mathrm{C}$ for $1 \mathrm{~min}, 35$ cycles of denaturation at $94{ }^{\circ} \mathrm{C}$ for $45 \mathrm{~s}$, annealing at $56{ }^{\circ} \mathrm{C}(\mathrm{CuZn}-\mathrm{SOD})$ or $55^{\circ} \mathrm{C}$ (CAT) for $1 \mathrm{~min}$, extension at $72{ }^{\circ} \mathrm{C}$ for $1 \mathrm{~min}$, and final extension at $72{ }^{\circ} \mathrm{C}$ for $15 \mathrm{~min}$. The protocol for GAPDH was the same except for annealing at $65{ }^{\circ} \mathrm{C}$ for $1 \mathrm{~min}$ and amplification for 25 cycles. The PCR products were electrophoresed in a $2 \%$ agarose gel and stained with ethidium bromide. The bands were automatically analyzed by Smart View Bioelectrophoresis Image Analysis system (Version FR-980, FURI Science and Technology Co. Ltd., Shanghai, China).

\subsection{Statistical analysis}

All experimental data were expressed as mean \pm standard error of mean (SEM). Significant differences among experimental groups were compared by one-way analysis of variance (ANOVA) followed by least significant difference (LSD) $(P<0.05)$ using the Statistics Package for Social Science (SPSS) program Version 11.5.

\section{Results}

\subsection{Ferulic acid prevents diosbulbin B-induced liver injury}

Serum ALT and AST activities are liver injury biomarkers, a significant elevation of which often reflects liver injury (Kamei et al., 1986). In the present study, ALT and AST were both found to be raised in mice treated with diosbulbin B alone for 12 consecutive days compared with CMC-Na controls $(P<0.001)$, while ferulic acid inhibited this diosbulbin B-induced increase $(P<0.001)$ (Fig. 2).
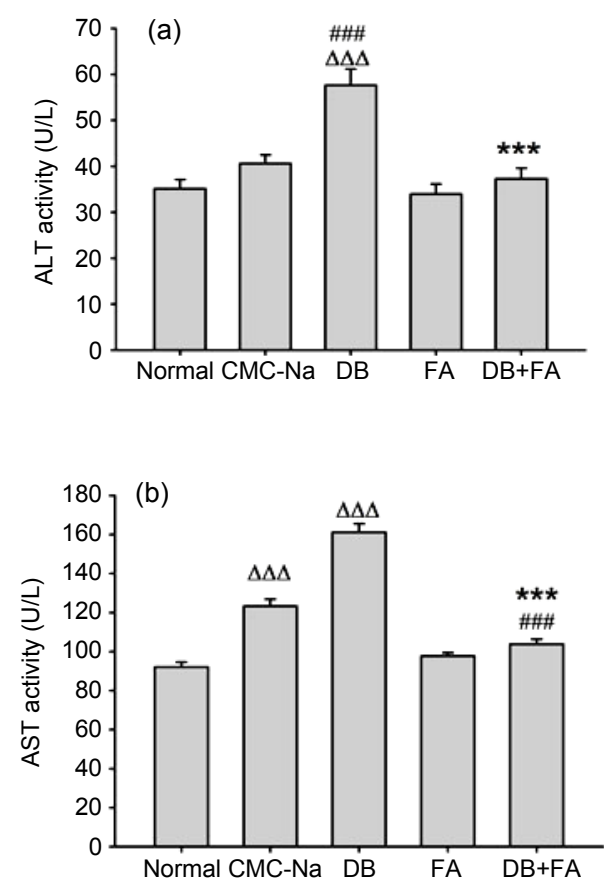

Fig. 2 Effect of ferulic acid (FA) on ALT (a) and AST (b) activities

FA inhibited diosbulbin B (DB)-induced increase of ALT/AST in tumor-bearing mice. Data are presented as mean \pm SEM $(n=8) .{ }^{\Delta 4} P<0.001$ vs. normal; ${ }^{\# \#} P<0.001$ vs. CMC-Na; ${ }^{* * *} P<0.001$ vs. DB

As shown in Fig. 3, malondialdehyde, one of the main end-products of lipid peroxide activity (Nkosi et al., 2006), was increased in the livers of mice treated with diosbulbin B compared with CMC-Na controls $(P<0.05)$, while ferulic acid inhibited this increase $(P<0.001)$.

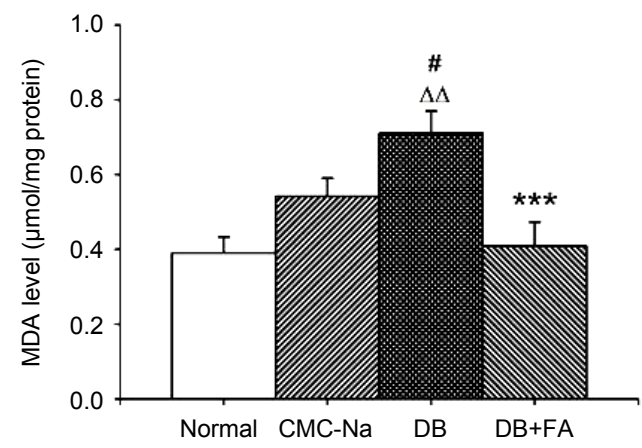

Fig. 3 Effect of ferulic acid (FA) on malondialdehyde (MDA) level

FA inhibited diosbulbin B (DB)-increased MDA amount. Data are presented as mean \pm SEM $(n=8) .{ }^{\Delta \Lambda} P<0.01$ vs. normal; ${ }^{\#} P<0.05$ vs. CMC-Na; ${ }^{* * *} P<0.001$ vs. $\mathrm{DB}$ 
Further, histological evaluation of the livers removed from mice treated with diosbulbin B (32 $\mathrm{mg} / \mathrm{kg}$ ) showed hydropic degeneration of hepatocytes (Fig. 4c). After treatment with ferulic acid $(8 \mathrm{mg} / \mathrm{kg})$, these abnormal changes disappeared (Fig. 4d).

\subsection{Ferulic acid reverses diosbulbin B-decreased CuZn-SOD and CAT enzymatic activities and mRNA expression}

CAT and CuZn-SOD are both important intracellular anti-oxidant enzymes, participating in the process of oxidative stress (Aebi, 1984; Zelko et al.,
2002). Our results showed that diosbulbin B decreased the enzymatic activities of CuZn-SOD and CAT compared with CMC-Na controls $(P<0.05$ and $P<0.01$, respectively), while ferulic acid reversed this decrease (both $P<0.01$ ) (Figs. $5 \mathrm{a}$ and $5 \mathrm{~b}$ ). Further results (Fig. 5c) showed that in the CMC-Na control group, mRNA expressions of CAT and $\mathrm{CuZn}-\mathrm{SOD}$ were decreased compared with the normal $(P<0.05$ and $P<0.001$, respectively). The mRNA expressions of CAT and CuZn-SOD in diosbulbin B-treated mice were further decreased compared with the CMC-Na group (both $P<0.01$ ), while ferulic acid clearly reversed this decrease $(P<0.05$ and $P<0.01$, respectively).
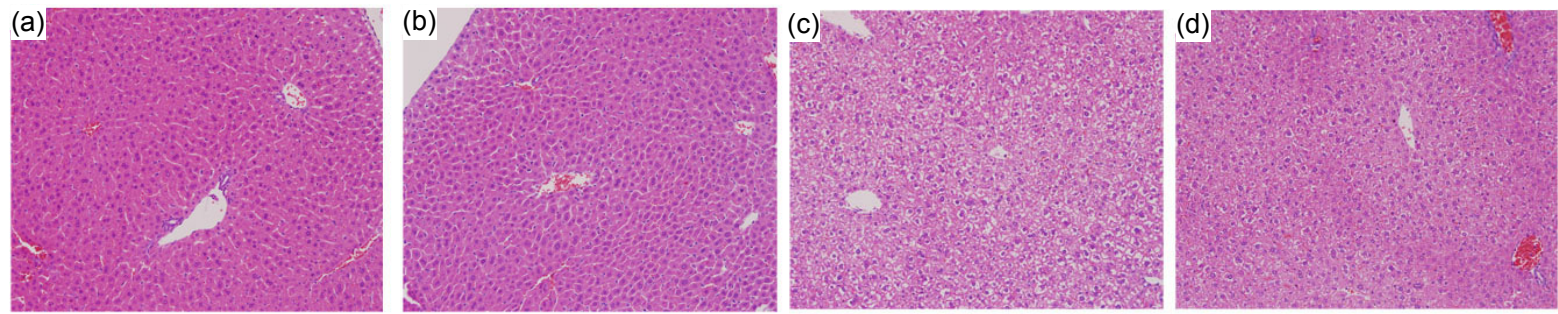

Fig. 4 Liver histology of the normal (a), CMC-Na control (b), diosbulbin B (32 mg/kg) (c), and diosbulbin B (32 $\mathrm{mg} / \mathrm{kg})+$ ferulic acid $(8 \mathrm{mg} / \mathrm{kg})(\mathrm{d})$ groups

After treatment, livers were removed, fixed, sectioned $(5 \mu \mathrm{m})$, and processed for H\&E staining. Typical images were chosen from each experimental group (original magnification $200 \times$ )

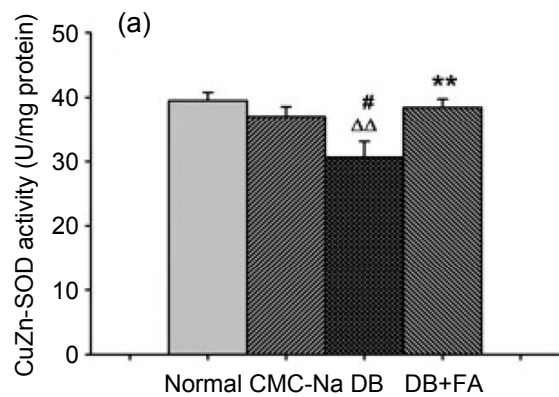

(c)
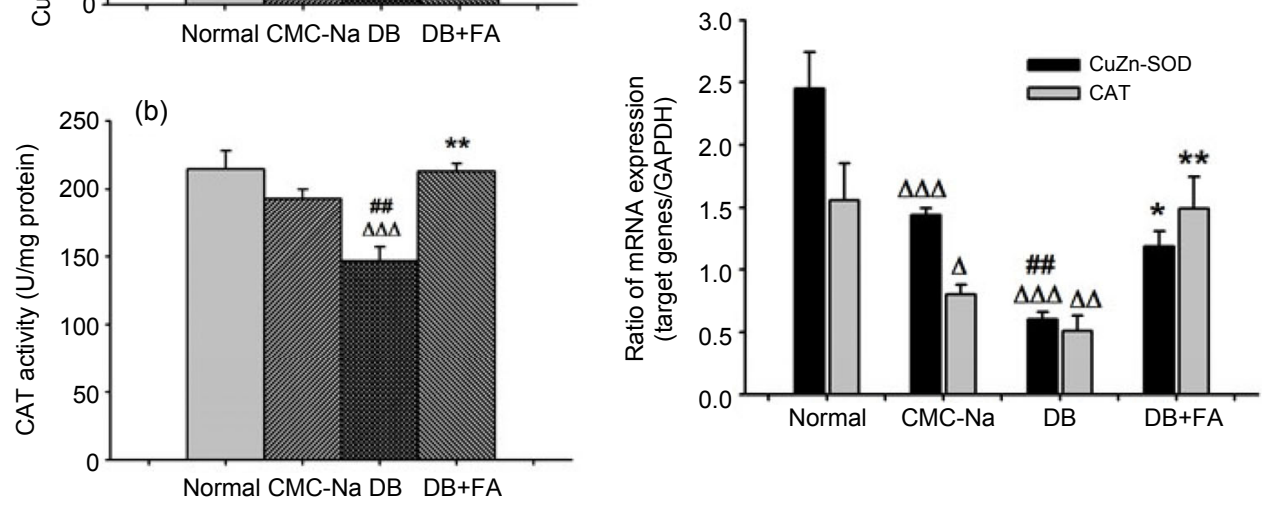

Fig. 5 Effects of ferulic acid (FA) on CuZn-SOD and CAT enzymatic activity and mRNA expression FA reversed diosbulbin B (DB)-decreased liver CuZn-SOD (a) and CAT (b) enzymatic activity and mRNA expression (c). Data are presented as mean \pm SEM, with $n=8$ (a, b) or $n=6$ (c). ${ }^{\Delta} P<0.05,{ }^{\Delta \Lambda} P<0.01,{ }^{\Delta \Lambda \Delta} P<0.001$ vs. normal; ${ }^{\#} P<0.05$, ${ }^{\#} P<0.01$ vs. CMC-Na; ${ }^{*} P<0.05,{ }^{* * *} P<0.01$ vs. DB 


\subsection{Ferulic acid enhances diosbulbin B-induced anti-tumor activity}

The effect of ferulic acid on the anti-tumor activity of diosbulbin B in transplanted S180 sarcoma is shown in Fig. 6. Our results showed that diosbulbin B decreased tumor weight of tumor-bearing mice $(P<0.01)$ by $51.8 \%$. After treatment with ferulic acid, diosbulbin Bdecreased tumor weight was further decreased by $75.5 \%$ compared with diosbulbin $\mathrm{B}$ alone $(P<0.01)$. Meanwhile, ferulic acid itself had no demonstrable inhibition of tumor growth.

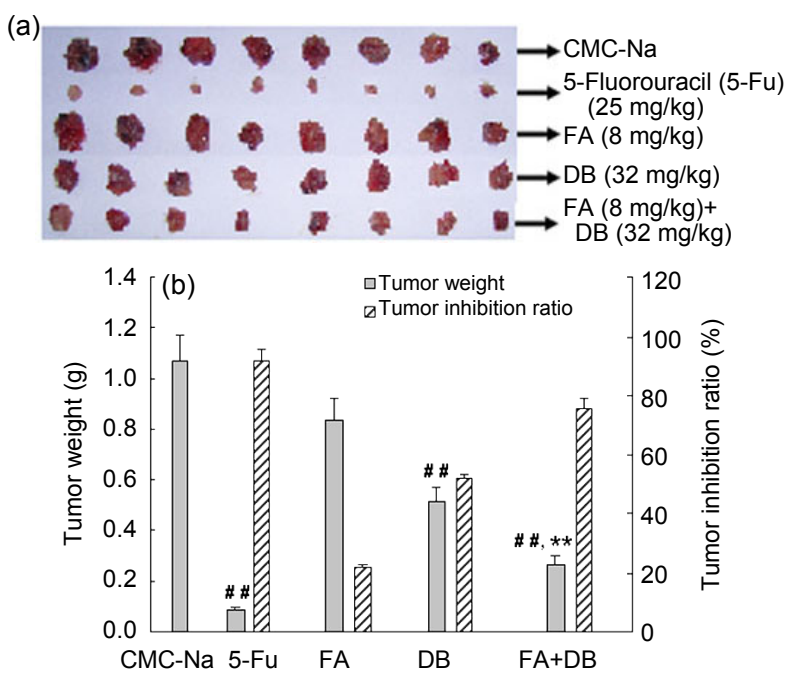

Fig. 6 Effect of ferulic acid (FA) on anti-tumor activity FA augmented diosbulbin B (DB)-induced anti-tumor activity in vivo. After treatment, the tumors were excised and weighed. (a) Pictures of tumor; (b) Tumor weight and the inhibitory ratio of tumor growth. Data are presented as mean \pm SEM $(n=8) .{ }^{\#} P<0.01$ vs. $\mathrm{CMC}-\mathrm{Na} ;{ }^{* *} P<0.01$ vs. DB

\section{Discussion}

Much attention has been paid to the prevention of cancer (Glade, 1999). In addition to western therapies for cancer prevention, there are also studies on anti-tumor effects of herbal medicines such as $D$. bulbifera, Tripterygium wilfordii, and Andrographis paniculata (Shamon et al., 1997; Gao et al., 2002; Kumar et al., 2004). Compatibility is a main principle of Chinese medicinal herbs, and some reports have demonstrated that compatibility may lead to eliminating toxicity or reinforcing bioactivities (Kim et al., 2007; Gao et al., 2009). Ferulic acid is the main bioactive component of $A$. sinensis root, and our results from ALT/AST assays and histological observations are the first to demonstrate that ferulic acid prevents diosbulbin B-induced liver injury. Furthermore, the results show that ferulic acid enhances the diosbulbin Binduced anti-tumor effect. All these results indicate that the compatibility of $A$. sinensis root with $D$. bulbifera may relieve the hepatotoxicity caused by $D$. bulbifera, and provide the possibility of clinical applications of these two drugs for cancer therapy.

The imbalance of oxidants and anti-oxidants will lead to oxidative stress in the liver. Further, many anti-oxidant enzymes and non-enzymatic anti-oxidants may be changed during this process (Łuczaj and Skrzydlewska, 2004; Dadkhah et al., 2006; LimónPacheco and Gonsebatt, 2009). Among them, lipid peroxide is free radical-related (Romero et al., 1998), and one of the main end-products of lipid peroxide is malondialdehyde. The results show that ferulic acid inhibited the diosbulbin B-induced increase in malondialdehyde, indicating that ferulic acid can prevent diosbulbin B-induced lipid peroxide injury in the liver.

CAT and SOD are believed to play key roles in the enzymatic defense of cells against oxidative stress injury. CAT mainly exists in the peroxisomes of aerobic cells and serves to protect against damage from hydrogen peroxide by catalyzing it into molecular oxygen and water without producing toxic free radicals (Bocchetti and Regoli, 2006; Yilmaz et al., 2006). The metalloenzyme, SOD, can convert two molecules of superoxide, produced during the oxidative stress, to hydrogen peroxide (Bocchetti and Regoli, 2006). There are three SOD isoenzymes in mammalian cells: CuZn-SOD (copper- and zinc-containing SOD, mainly cytosolic), Mn SOD (manganese-containing SOD, located in the mitochondria), and EC SOD (extracellular SOD, actually also CuZn-SOD) (Zelko et al., 2002); of these, CuZn-SOD is probably the most important anti-oxidant enzyme (Peskin, 1997). Our results showed that ferulic acid reversed diosbulbin Bdecreased enzymatic activities and mRNA expressions of CAT and CuZn-SOD. The results suggest that ferulic acid can prevent diosbulbin B-induced oxidative stress injury, while CAT and CuZn-SOD may also participate in this protection.

In conclusion, the present study shows that ferulic acid can prevent hepatotoxicity induced by diosbulbin B isolated from D. bulbifera rhizome, 
mainly via preventing liver oxidative stress injury. This study also demonstrates that ferulic acid can enhance the anti-tumor activity of diosbulbin $\mathrm{B}$ in mice. Further studies are in progress in our laboratory to explore the molecular mechanisms of this reinforced anti-tumor activity.

\section{Compliance with ethics guidelines}

Jun-ming WANG, Yu-chen SHENG, Li-li JI, and Zhengtao WANG declare that they have no conflict of interest.

All institutional and national guidelines for the care and use of laboratory animals were followed.

\section{References}

Aebi, H., 1984. Catalase in vitro. Methods Enzymol., 105: 121-126. [doi:10.1016/S0076-6879(84)05016-3]

Alias, L.M., Manoharan, S., Vellaichamy, L., et al., 2009. Protective effect of ferulic acid on 7,12-dimethylbenz[a] anthracene-induced skin carcinogenesis in Swiss albino mice. Exp. Toxicol. Pathol., 61(3):205-214. [doi:10.1016/ j.etp.2008.09.001]

Barone, E., Calabrese, V., Mancuso, C., 2009. Ferulic acid and its therapeutic potential as a hormetin for age-related diseases. Biogerontology, 10(2):97-108. [doi:10.1007/ s10522-008-9160-8]

Bezerra, D.P., Pessoa, C., Moraes, M.O., et al., 2008. In vivo growth-inhibition of sarcoma 180 by piperlonguminine, an alkaloid amide from the Piper species. J. Appl. Toxicol., 28(5):599-607. [doi:10.1002/jat.1311]

Bocchetti, R., Regoli, F., 2006. Seasonal variability of oxidative biomarkers, lysosomal parameters, metallothioneins and peroxisomal enzymes in the Mediterranean mussel Mytilus galloprovincialis from Adriatic Sea. Chemosphere, 65(6):913-921. [doi:10.1016/j.chemosphere.2006. 03.049]

Calabrese, V., Calafato, S., Puleo, E., et al., 2008. Redox regulation of cellular stress response by ferulic acid ethyl ester in human dermal fibroblasts: role of vitagenes. Clin. Dermatol., 26(4):358-363. [doi:10.1016/j.clindermatol. 2008.01.005]

Dadkhah, A., Fatemi, F., Kazemnejad, S., et al., 2006. Differential effects of acetaminophen on enzymatic and non-enzymatic antioxidant factors and plasma total antioxidant capacity in developing and adult rats. Mol. Cell. Biochem., 281(1-2):145-152. [doi:10.1007/s11010-0060719-x]

dos Santos, N.A., Martins, N.M., Curti, C., et al., 2007. Dimethylthiourea protects against mitochondrial oxidative damage induced by cisplatin in liver of rats. Chem. Biol. Inter., 170(3):177-186. [doi:10.1016/j.cbi.2007.07.014]

El Mouatassim, S., Guérin, P., Ménézo, Y., 1999. Expression of genes encoding antioxidant enzymes in human and mouse oocytes during the final stages of maturation. $\mathrm{Mol}$. Hum. Reprod., 5(8):720-725. [doi:10.1093/molehr/5.8.720]

Fetoni, A.R., Mancuso, C., Eramo, S.L., et al., 2010. In vivo protective effect of ferulic acid against noise-induced hearing loss in the guinea-pig. Neuroscience, 169(4):
1575-1588. [doi:10.1016/j.neuroscience.2010.06.022]

Gao, H., Kuroyanagi, M., Wu, L., et al., 2002. Antitumorpromoting constituents from Dioscorea bulbifera L. in JB6 mouse epidermal cells. Biol. Pharm. Bull., 25(9): 1241-1243. [doi:10.1248/bpb.25.1241]

Gao, J.L., He, T.C., Li, Y.B., et al., 2009. A traditional Chinese medicine formulation consisting of Rhizoma corydalis and Rhizoma curcumae exerts synergistic anti-tumor activity. Oncol. Rep., 22(5):1077-1083. [doi:10.3892/or 00000539]

Glade, M.J., 1999. Food, nutrition, and the prevention of cancer: a global perspective (American Institute for Cancer Research/World Cancer Research Fund, American Institute for Cancer Research, 1997). Nutrition, 15(6): 523-526. [doi:10.1016/S0899-9007(99)00021-0]

Graf, E., 1992. Antioxidant potential of ferulic acid. Free Radical Biol. Med., 13(4):435-448. [doi:10.1016/08915849(92)90184-I]

Hougardy, B.M., van der Zee, A.G., van den Heuvel, F.A., et al., 2005. Sensitivity to Fas-mediated apoptosis in high-risk HPV-positive human cervical cancer cells: relationship with Fas, caspase-8, and Bid. Gynecol. Oncol., 97(2): 353-364. [doi:10.1016/j.ygyno.2005.01.036]

Kamei, T., Asano, K., Nakamura, S., 1986. Determination of serum glutamate oxaloacetate transaminase and glutamate pyruvate transaminase by using L-glutamate oxidase. Chem. Pharm. Bull., 34(1):409-412. [doi:10.1248/cpb. 34.409]

Kawasaki, T., Komori, T., Setoguchi, S., 1968. Furanoid norditerpenes from Dioscoreacae plants. 1. Diosbulins A, $\mathrm{B}$, and C from Dioscorea bulbifera form a spontanea. Chem. Pharm. Bull., 16(12):2430-2435. [doi:10.1248/cpb. 16.2430]

Kim, K.C., Kim, J.S., Son, J.K., et al., 2007. Enhanced induction of mitochondrial damage and apoptosis in human leukemia HL-60 cells by the Ganoderma lucidum and Duchesnea chrysantha extracts. Cancer Lett., 246(1-2): 210-217. [doi:10.1016/j.canlet.2006.02.014]

Kostopanagiotou, G.G., Grypioti, A.D., Matsota, P., et al., 2009. Acetaminophen-induced liver injury and oxidative stress: protective effect of propofol. Eur. J. Anaesthesiol., 26(7):548-553. [doi:10.1097/EJA.0b013e32831c8a01]

Kumar, R.A., Sridevi, K., Kumar, N.V., et al., 2004. Anticancer and immunostimulatory compounds from $\mathrm{An}$ drographis paniculata. J. Ethnopharmacol., 92(2-3): 291-295. [doi:10.1016/j.jep.2004.03.004]

Limón-Pacheco, J., Gonsebatt, M.E., 2009. The role of antioxidants and antioxidant-related enzymes in protective responses to environmentally induced oxidative stress. Mutat. Res./Genet. Toxicol. Environ. Mutagen., 674(1-2): 137-147. [doi:10.1016/j.mrgentox.2008.09.015]

Liu, H., Chou, G.X., Guo, Y.L., et al., 2010. Norclerodane diterpenoids from rhizomes of Dioscorea bulbifera. Phytochemistry, 71(10):1174-1180. [doi:10.1016/j.phytochem. 2010.04.002]

Liu, S.M., Li, Y.J., Luo, M.M., et al., 2004. The detoxification action of Chinese Angelica root to Dioscorea bulbifera L. Chin. J. Integr. Trad. West. Med. Liver Dis., 14(4): 216-218 (in Chinese). 
Łuczaj, W., Skrzydlewska, E., 2004. Antioxidant properties of black tea in alcohol intoxication. Food Chem. Toxicol., 42(12):2045-2051. [doi:10.1016/j.fct.2004.08.009]

Marklund, S.L., Marklund, G., 1974. Involvement of the superoxide anion radical in the autoxidation of pyrogallol and a convenient assay for superoxide dismutase. Eur. $J$. Biochem., 47(3):469-474. [doi:10.1111/j.1432-1033.1974. tb03714.x]

Moselhy, S.S., Ali, H.K., 2009. Hepatoprotective effect of cinnamon extracts against carbon tetrachloride induced oxidative stress and liver injury in rats. Biol. Res., 42(1): 93-98. [doi:10.4067/S0716-97602009000100009]

Murray, R.D.H., Jorge, Z., Khan, N.H., et al., 1984. Diosbulbin $\mathrm{D}$ and 8 -epidiosbulbin $\mathrm{E}$ acetate, norclerodane diterpenoids from Dioscorea bulbifera tubers. Phytochemistry, 23(3):623-625. [doi:10.1016/S0031-9422(00) 80394-5]

Nkosi, C.Z., Opoku, A.R., Terblanche, S.E., 2006. Antioxidative effects of pumpkin seed (Cucurbita pepo) protein isolate in $\mathrm{CCl}_{4}$-induced liver injury in low-protein fed rats. Phytother. Res., 20(11):935-940. [doi:10.1002/ptr.1977]

Peskin, A.V., 1997. Cu, Zn-superoxide dismutase gene dosage and cell resistance to oxidative stress: a review. Biosci. Rep., 17(1):85-89. [doi:10.1023/A:1027343519591]

Romero, F.J., Bosch-Morell, F., Romero, M.J., et al., 1998. Lipid peroxidation products and antioxidants in human disease. Environ. Health Perspect., 106(Suppl. 5):12291234. [doi:10.1289/ehp.98106s51229]

Samuhasaneeto, S., Thong-Ngam, D., Kulaputana, O., et al., 2009. Curcumin decreased oxidative stress, inhibited $\mathrm{NF}-\mathrm{\kappa B}$ activation, and improved liver pathology in ethanolinduced liver injury in rats. J. Biomed. Biotechnol., 2009:981963. [doi:10.1155/2009/981963]

Shamon, L.A., Pezzuto, J.M., Graves, J.M., et al., 1997. Evaluation of the mutagenic, cytotoxic, and antitumor potential of triptolide, a highly oxygenated diterpene isolated from Tripterygium wilfordii. Cancer Lett., 112(1): 113-117. [doi:10.1016/S0304-3835(96)04554-5]

Sun, Y., Li, S., Song, H., et al., 2006. Extraction of ferulic acid from Angelica sinensis with supercritical $\mathrm{CO}_{2}$. Nat. Prod. Res., 20(9):835-841. [doi:10.1080/14786410500462579]

van der Vliet, A., O'Neill, C.A., Cross, C.E., et al., 1999. Determination of low-molecular-mass antioxidant concentrations in human respiratory tract lining fluids. $\mathrm{Am}$. $\mathrm{J}$. Physiol., 276(2 Pt 1):289-296.

Wang, G., Liu, J.S., Lin, B.B., et al., 2009. Two new furanoid norditerpenes from Dioscorea bulbifera. Chem. Pharm. Bull., 57(6):625-627. [doi:10.1248/cpb.57.625]

Wang, J., Ji, L., Liu, H., et al., 2010. Study of the hepatotoxicity induced by Dioscorea bulbifera L. rhizome in mice. Biosci. Trends, 4(2):79-85.

Wang, J., Liang, Q., Ji, L., et al., 2011. Gender-related difference in liver injury induced by Dioscorea bulbifera L. rhizome in mice. Hum. Exp. Toxicol., 30(9):1333-1341. [doi:10.1177/0960327110389926]

Wang, J., Ji, L., Branford-White, C.J., et al., 2012. Antitumor activity of Dioscorea bulbifera L. rhizome in vivo. Fitoterapia, 83(2):388-394. [doi:10.1016/j.fitote.2011. 12.001]

Yilmaz, H.R., Turkoz, Y., Yuksel, E., et al., 2006. An investigation of antioxidant enzymes activities in liver of $C y$ prinus carpio taken from different stations in the Karakaya Dam Lake. Int. J. Food Sci. Tech., 1(1):1-6.

You, Y., Park, J., Yoon, H.G., et al., 2009. Stimulatory effects of ferulic acid on endurance exercise capacity in mice. Biosci. Biotechnol. Biochem., 73(6):1392-1397. [doi:10. 1271/bbb.90062]

Zelko, I.N., Mariani, T.J., Folz, R.J., 2002. Superoxide dismutase multigene family: a comparison of the CuZn-SOD (SOD1), Mn-SOD (SOD2), and EC-SOD (SOD3) gene structures, evolution, and expression. Free Radical Biol. Med., 33(3):337-349. [doi:10.1016/S0891-5849(02)00 905-X]

\section{中文概要:}

本文题目：阿魏酸降低黄独素 B 肝毒性并提高其抗肿瘤活性

Ferulic acid prevents liver injury and increases the anti-tumor effect of diosbulbin $B$ in vivo

研究目的: 观察阿魏酸对黄独素 B 诱导肝毒性的抑制活性及其机理, 同时探索阿魏酸对黄独素 B 抗肿瘤 活性的增效作用。

创新要点: 黄独素 B 为中药黄药子抗肿瘤的主要药效活性成分, 但同时又是其致肝毒性的主要毒性成分。 本研究立足于中医药配伍减毒增效理论, 试图通过试验考察配伍阿魏酸对黄独素 B 肝毒性/ 抗肿瘤活性的减毒增效作用, 为黄独素 B 与阿魏酸联合应用于抗肿瘤提供了一定的临床前试 验依据。

研究方法: 荷瘤小鼠 (S180 肉瘤) 连续 12 天灌胃给药阿魏酸和黄独素 B。通过对血清丙氨酸/天冬氨酸 转氨酶活性（见图 2)、肝脂质过氧化（见图 3) 和肝组织病理分析（见图 4) 考察阿魏酸对 黄独素 B 肝毒性的抑制作用；通过对铜锌-超氧化物歧化酶和过氧化氢酶的活性（见图 5a、 $5 \mathrm{~b}$ ）和基因表达分析（见图 5c）探讨阿魏酸抑制黄独素 B 肝毒性的机理；通过对瘤重、抑 瘤率的统计分析阿魏酸增加的黄独素 B 抗肿瘤活性。

重要结论: 阿魏酸可以通过改善黄独素 B 诱导的氧应激损伤从而抑制其肝毒性, 同时还可以协同增加黄 独素 B 的抗肿瘤活性。

关键词组: 阿魏酸; 黄独素 B; 肝毒性; 氧应激损伤; 抗肿瘤 